\title{
Comparação entre as análises auditiva e acústica nas disartrias
}

\section{Comparison between auditory-perceptual and acoustic analyses in dysarthrias}

\author{
Karin Zazo Ortiz ${ }^{1}$, Luciane Carrillo ${ }^{2}$
}

\begin{abstract}
RESUMO
Objetivo: Comparar os dados da análise perceptivo-auditiva (subjetiva) com os dados da análise acústica (objetiva). Métodos: Quarenta e dois pacientes disártricos, com diagnósticos neurológicos definidos, 21 do sexo masculino e 21 do sexo feminino foram submetidos à análise perceptual-auditiva e acústica. Todos os pacientes foram submetidos à gravação da voz, tendo sido avaliados, na análise auditiva, tipo de voz, ressonância (equilibrada, hipernasal ou laringo-faríngea), loudness (adequado, diminuído ou aumentado), pitch (adequado, grave, agudo) ataque vocal (isocrônico, brusco ou soproso), e estabilidade (estável ou instável). Para a análise acústica foram utilizados os programas GRAM 5.1.7; para a análise da qualidade vocal e comportamento dos harmônicos na espectrografia e o Programa Vox Metria, para a obtenção das medidas objetivas. Resultados: A comparação entre os achados das análises auditiva e acústica em sua maioria não foi significante, ou seja, não houve uma relação direta entre os achados subjetivos e os dados objetivos. Houve diferença estatisticamente significante apenas entre voz soprosa e Shimmer alterado ( $\mathrm{p}=0,048)$ e entre a definição dos harmônicos e voz soprosa ( $\mathrm{p}=0,040)$, sendo assim, observou-se correlação entre a presença de ruído à emissão e soprosidade. Conclusões: As análises perceptual-auditiva e acústica forneceram dados diferentes, porém complementares, auxiliando, de forma conjunta, no diagnóstico clínico das disartrias.
\end{abstract}

Descritores: Disartria; Distúrbios da voz; Acústica da fala; Fala; Qualidade da voz

\section{INTRODUÇÃO}

A disartria é um nome coletivo para as alterações de fala resultantes de distúrbios no controle de seu mecanismo, devido a danos no sistema nervoso central ou periférico; designa problemas na comunicação oral devido à paralisia, fraqueza ou incoordenação da musculatura da fala. Ocorre como um déficit na produção da fala resultante de alteração no sistema nervoso central ou periférico que afeta um ou vários dos cinco componentes da produção da fala: respiração, fonação, ressonância, articulação e prosódia ${ }^{(1-2)}$.

As disfonias neurológicas são comuns nas disartrias e desempenham um importante papel no diagnóstico diferencial. Portanto, é essencial que a avaliação e a terapia de voz

Trabalho realizado no Departamento de Fonoaudiologia da Universidade Federal de São Paulo - UNIFESP - São Paulo (SP), Brasil, com financiamento da Fundação de Amparo à Pesquisa do Estado de São Paulo (FAPESP), processo 04/09950-2.

(1) Doutora, Professora do Departamento de Fonoaudiologia da Universidade Federal de São Paulo - UNIFESP - São Paulo (SP), Brasil.

(2) Especialista em Voz pelo Centro de Estudos da Voz - CEV - São Paulo (SP), Brasil; Pós-graduanda do Communication Studies Department da San Jose State University - SJSU - San Jose (CA), Estados Unidos.

Endereço para correspondência: Karin Zazo Ortiz. R. Cunha, 111, cjto 73, Vila Clementino, São Paulo - SP, CEP 04037-030. E-mail: karinortiz.fono@epm.br

Recebido em: 22/10/2007; Aceito em: 30/8/2008 considerem todo o mecanismo da fala: respiração, fonação, articulação, ressonância e prosódia.

A coexistência de alteração dos diversos subsistemas deve levar o clínico a dirigir a avaliação fonoaudiológica para testes específicos de exploração de aspectos neurológicos da emissão do indivíduo. Voz rouca, geralmente é uma queixa comum trazida pelo paciente ao médico e/ou fonoaudiólogo. A natureza da queixa é vaga e subjetiva e podem existir múltiplos fatores etiológicos que causam o transtorno vocal.

Dependendo da localização da lesão neurológica têm-se manifestações diversas na fala, voz e linguagem do indivíduo. Os distúrbios neurológicos da fala podem ser definidos de acordo com o nível anatômico afetado: transtornos do neurônio motor superior (esclerose lateral amiotrófica - ELA disartria mista e paralisia pseudobulbar - disartria espástica); transtornos do neurônio motor inferior (ELA - disartria mista e paralisia bulbar - disartria flácida); transtornos do sistema extrapiramidal (doença de Parkinson - disartria hipocinética, distonia - disartria hipercinética lenta e Coréa - disartria hipercinética rápida); transtornos do cerebelo (doença cerebelar degenerativa - disatria atáxica, ataxia hereditária, transtornos metabólicos tóxicos, lesões vasculares e esclerose múltipla - disartria mista, espástica e atáxica); transtornos da junção neuromuscular e transtornos mistos (disfonia espástica) ${ }^{(3)}$.

Lesões cerebrais no lado esquerdo parecem mostrar impacto mais severo na articulação, afetando principalmente a 
produção das consoantes, independente da lesão topográfica. No entanto, alterações na fonação e prosódia também são freqüentes ${ }^{(4)}$.

As disfonias associadas às doenças neurológicas geralmente contribuem substancialmente para a severidade do quadro geral, e muitas vezes são consideradas pelos pacientes e familiares como um dos principais fatores responsáveis pela depressão e isolamento social característico de determinadas doenças.

A avaliação fonoaudiológica de um paciente disártrico deve ser minuciosa e integrar os achados otorrinolaringológicos e neurológicos, a fim de contar com o máximo de informações para traçar um plano terapêutico efetivo maximizando os resultados para o tratamento do paciente.

A avaliação da função fonatória nas disartrias é clinicamente importante ante uma série de razões. Primeiramente, os distúrbios fonatórios têm um papel de destaque no estabelecimento do diagnóstico diferencial. A fonação normal requer um controle motor extremamente precioso. Qualquer fraqueza, lentidão ou incoordenação da musculatura laríngea pode ser percebida através da voz. A identificação de sinais e sintomas laríngeos, portanto, pode auxiliar no diagnóstico diferencial precoce de algumas doenças. Nos casos mais severos dos distúrbios neurológicos, o exame das características fonatórias pode revelar informações importantes sobre a neuropatologia subjacente. Além disso, a compreensão total dos aspectos fonatórios das disartrias também é de crucial valor no planejamento terapêutico, pois, às vezes, a alteração isolada de um parâmetro pode limitar a comunicação funcional do indivíduo.

Uma vez que as queixas dos indivíduos disártricos raramente se remetem a alterações vocais específicas, como, por exemplo, rouquidão, hipernasalidade etc., mas sim à efetividade da comunicação social, inicialmente o paciente deve ser avaliado quanto às características de naturalidade e inteligibilidade de sua comunicação, ou seja, o quanto ela pode ser considerada efetiva ou não. Um dos parâmetros essenciais para o diagnóstico e a reabilitação é a qualidade vocal.

Em nosso meio, a avaliação de um paciente com alteração vocal neurológica ainda é essencialmente auditiva, mas deveria ser complementada por análise acústica e métodos fisiológicos de avaliação vocal e laríngea.

Nos últimos anos, tem-se aplicado a análise acústica e fisiológica em pacientes com doenças neurológicas. A análise acústica propicia mensurações do sinal sonoro vocal, enquanto que a avaliação perceptivo-auditiva oferece uma descrição do sinal vocal tendo como instrumento básico a audição ${ }^{(5)}$.

A análise acústica da voz de um paciente que sofreu uma lesão neurológica é uma importante ferramenta na detecção precoce, diagnóstico diferencial e monitoramento de uma doença progressiva. Embora a análise perceptiva das vozes patológicas contribua para esses objetivos, a análise acústica tem permitido dados quantitativos e sensíveis do funcionamento fonatório ${ }^{(6)}$.

$\mathrm{Na}$ análise acústica, a avaliação global do traçado espectrográfico nos traz uma informação detalhada sobre a fonte sonora e o sistema de ressonância, representando uma verdadeira reprodução gráfica da qualidade vocal. Já a distribuição dos harmônicos no espectro (harmônicos superiores e ruído entre os harmônicos) nos oferece indicação simples e direta sobre a ressonância e a projeção vocal do paciente. Quanto maior a série de harmônicos identificada, mais rica é a qualidade vocal. Além disso, quanto mais individualizados estão os harmônicos unitários, e quanto mais linear é o traçado (definição dos harmônicos e estabilidade), maior é o componente harmônico e a estabilidade na sustentação. A presença de um traçado irregular, retorcido, interrompido e uma imagem de enovelamento semelhante a um borrão, são indicativos de aumento do componente ruído na emissão. Além disso, na análise acústica quantitativa os seguintes parâmetros: $\mathrm{F}_{0}$ (frequiência fundamental) e seus índices de perturbação (Jitter e Shimmer) oferecem dados sobre a similaridade dos ciclos glóticos sucessivos e sobre a estabilidade da fonte glótica. A $\mathrm{F}_{0}$ correspondente ao número de ciclos vibratórios nas pregas vocais a cada segundo; o Jitter indica a variabilidade da frequiência fundamental a curto prazo; o Shimmer indica a variabilidade da amplitude da onda sonora a curto prazo e é uma medida de estabilidade fonatória; o GNE - Glottal to Noise Excitation ratio é uma medida acústica para calcular o ruído em uma série de pulsos produzidos pela oscilação das pregas vocais. Esse parâmetro é baseado na hipótese de que pulsos resultantes da colisão das pregas vocais geram uma excitação síncrona de diferentes faixas de frequiência. Por outro lado, o ruído produzido pelas pregas vocais comprimidas gera excitações não correlacionadas. Um valor próximo a um significa uma excitação do tipo "pulso" (ex: uma excitação normal da glote), enquanto valores próximos a zero resultam de uma excitação do tipo ruído (ex: voz sussurrada). O diagrama de desvio fonatório é um gráfico que demonstra o comportamento vocal, sendo que a posição no eixo horizontal indica as medidas de Jitter e Shimmer e a posição no eixo vertical, medidas da quantidade de ruído - GNE(7).

Considerando-se o exposto, esta pesquisa suporta a noção de que a análise acústica pode prover características qualitativas e quantitativas da voz, as quais podem ter relevância clínica, como também acadêmica, no campo das vozes patológicas, sendo importante para profissionais das áreas de fonoaudiologia, otorrinolaringologia e neurologia.

Neste trabalho, enfatizaram-se os diferentes aspectos dos distúrbios da fonação nas disfonias neurológicas, já que estes influenciam a inteligibilidade de fala e são clinicamente importantes para o diagnóstico e reabilitação. Desta forma, descrevemos clinica e objetivamente a voz nos diferentes tipos de disartrias e objetivamos compararmos os resultados da análise perceptivo-auditiva (subjetiva) com a acústica (objetiva) de pacientes disártricos com diferentes tipos de disartrias.

\section{MÉTODOS}

Este projeto foi aprovado pelo Comitê de Ética em Pesquisa da Universidade Federal de São Paulo sob o protocolo de número 517/04. Cada paciente foi convidado a participar da pesquisa e todos os participantes assinaram um termo de consentimento livre e esclarecido.

A amostra foi composta por 42 pacientes disártricos, que estavam em lista de espera para o atendimento no Ambulatório dos Distúrbios de Fala e Linguagem Adquiridos do Departa- 
mento de Fonoaudiologia da UNIFESP (Universidade Federal de São Paulo - Escola Paulista de Medicina). Todos os pacientes apresentavam diagnóstico neurológico etiológico definido, com idades variando de 16 a 80 anos. A amostra foi composta por pacientes do sexo masculino $(n=21)$ e feminino $(n=21)$.

Para a caracterização da amostra utilizou-se a definição das doenças neurológicas de acordo com o nível anatômico afetado $^{(3)}$. Dentre os transtornos do neurônio motor superior, a amostra foi composta por seis (14,3\% da amostra) pacientes com disartria espástica e um paciente com disartria mista (ELA) e cinco $(11,9 \%)$ pacientes com disartria do neurônio motor superior unilateral (NMSU); dentre os transtornos do neurônio motor inferior, oito (19\%) pacientes com disartria flácida; dentre os transtornos do sistema extrapiramidal, tivemos 14 (33,3\%) pacientes com doença de Parkinson; com disartria hipercinética lenta (distonia) tivemos sete $(16,7 \%)$ pacientes e um com disartria mista (miastenia gravis). Os pacientes com disartria mista representaram apenas $4,8 \%$ da amostra. Não houve na amostra, pacientes de transtornos do cerebelo. Pacientes desses grupos foram selecionados porque eles têm diferentes doenças neurológicas e disartrias que incluem alterações vocais. Podemos, no entanto, perceber que os grupos de pacientes não foram homogêneos, mas foram representativos dos encaminhamentos normalmente realizados pelos diferentes setores de neurologia da UNIFESP-EPM para $\mathrm{o}$ atendimento fonoaudiológico.

Todos os pacientes foram submetidos à gravação da voz, diretamente no computador, por ser o melhor meio de captação da voz para se realizar a análise acústica. Foi utilizado microfone Plantronix - modelo A-20. A gravação foi realizada em ambiente silencioso, com um distanciamento boca-microfone de $10 \mathrm{~cm}$, durante a emissão da vogal /é/ sustentada em condição habitual, contagem de números (de 1 a 10), repetição da frase "Um homem e uma mulher viram um anjo", depoimento sobre a própria voz e o canto da música "Parabéns a você".

Foram realizados três tipos de análises: análise auditiva, análise acústica e comparação entre análise auditiva e acústica.

A avaliação perceptivo-auditiva foi realizada por uma fonoaudióloga (com especialização em voz). Para tanto, foram analisadas as tarefas de vogal sustentada e contagem de números. Após a coleta dos dados, todas as gravações das vozes dos pacientes foram misturadas para a realização da análise auditiva, contabilizando 42 vozes, sendo que $10 \%$ da amostra foram apresentados novamente à avaliadora para confiabilidade dos dados.

Foram analisados seguintes parâmetros auditivos: tipo de voz, ressonância (equilibrada, hipernasal ou laringo-faríngea), loudness (adequado, diminuído ou aumentado); pitch (adequado, grave ou agudo), ataque vocal (isocrônico, brusco ou soproso) e estabilidade vocal (estável ou instável).

Para a análise acústica foram utilizados Programas GRAM 5.1.7., que permitiram analisar a qualidade vocal e o comportamento dos harmônicos na espectrografia, e o Programa Vox Metria, da CTS Informática, para a extração das medidas objetivas. Com estes dois programas obtivemos, além das medidas quantitativas, medidas qualitativas principalmente por meio de hipóteses perceptivas e observações ${ }^{(8-10)}$.
$\mathrm{Na}$ análise acústica foi avaliada a vogal /él. A tarefa de vogal sustentada foi selecionada porque facilita a interpretação da função fonatória por reduzir os efeitos da coarticulação, presentes na fala conectada, e por requerer o mínimo de treinamento do sujeito.

$\mathrm{Na}$ análise acústica qualitativa/ avaliação do comportamento dos harmônicos, foram analisados: traçado espectrográfico (estável ou instável), subharmônicos (presentes ou ausentes), harmônicos superiores (presentes ou ausentes), ruído entre os harmônicos (presente ou ausente), definição dos harmônicos (definidos ou mal definidos) e início da emissão (adequado, sem sonoridade ou com espículas).

$\mathrm{Na}$ análise acústica quantitativa foram avaliados os seguintes parâmetros: $\mathrm{F}_{0}$ (freqüência fundamental) e seus índices de perturbação (Jitter e Shimmer); medidas de ruído: GNE - Glottal to Noise Excitation ratio e diagrama de desvio fonatório. Neste parâmetro tivemos as variáveis, diagrama de desvio fonatório normal, alterado no sentido horizontal, alterado no sentido vertical e alterado no sentido horizontal e vertical.

Vale a pena ressaltar que o tempo máximo de fonação (TMF) é uma medida acústica, a de mais fácil obtenção, pois requer apenas um cronômetro. Foi utilizado cronômetro Radio Shack para extração das medidas. Foi extraído o TMF da vogal /é/.

Na comparação entre os dados da análise auditiva e da análise acústica, as variáveis foram diversas, estando apresentadas a seguir: tipo de voz (auditiva) X GNE (acústica); tipo de voz (auditiva) X subharmônicos (acústica); tipo de voz (auditiva) $\mathrm{X}$ ruído entre harmônicos (acústica); tipo de voz (auditiva) X definição dos harmônicos na espectrografia (acústica); tipo de voz (auditiva) X Jitter (acústica); voz trêmula (auditiva) X Jitter (acústica) e pitch (auditiva) X frequiência fundamental (acústica) - por sexo.

Os dados foram submetidos à análise estatística com os testes: teste Qui-quadrado (X2) (sem a correção de Yates), ou o teste exato de Fisher para comparações de dados categóricos; teste $t$ de Student para diferenças entre médias de dados contínuos e Mann Whitney (U) utilizado para comparação de dados contínuos não paramétricos entre duas amostras. Todas as análises foram calculadas segundo o pacote estatístico SPSS (Statistical Pakage for the Social Science) 11.5.1 para Windows. A probabilidade ( $\mathrm{p}$ ) menor que 0,05 foi considerada, para indicar significância estatística.

\section{RESULTADOS}

Os resultados são apresentados nas Tabelas 1 a 5.

\section{DISCUSSÃO}

A análise acústica computadorizada foi utilizada neste estudo, vindo ao encontro da filosofia de linhas atuais de pesquisa em voz, como uma das formas de buscar a comprovação objetiva, contribuir para a normatização dos dados para diferentes realidades vocais, bem como para o monitoramento da eficácia de diferentes procedimentos terapêuticos.

Inúmeras doenças neurológicas podem apresentar problemas de voz. Conseqüentemente, é essencial que profissionais que 
Tabela 1. Análise acústica qualitativa (traçado espectrográfico) nos diferentes tipos de disartria

\begin{tabular}{|c|c|c|c|c|}
\hline Tipo de disartria & $\begin{array}{l}\text { Estabilidade } \\
\text { do traçado }\end{array}$ & $\begin{array}{l}\text { Subharmônicos } \\
\text { (presença) }\end{array}$ & $\begin{array}{c}\text { Harmônicos superiores } \\
\text { (ausência) }\end{array}$ & $\begin{array}{l}\text { Presença de ruído } \\
\text { entre harmônicos }\end{array}$ \\
\hline Flácida & instável & $12 \%$ & $100 \%$ & $75 \%$ \\
\hline Espástica & instável & $33 \%$ & $100 \%$ & $100 \%$ \\
\hline Hipocinética & instável & $14 \%$ & $50 \%$ & $93 \%$ \\
\hline Hipercinética & instável & $14 \%$ & $85 \%$ & $86 \%$ \\
\hline NMSU & instável & $20 \%$ & $80 \%$ & $80 \%$ \\
\hline Mista & instável & $50 \%$ & $50 \%$ & $50 \%$ \\
\hline
\end{tabular}

Legenda: NMSU = Neurônio motor superior unilateral

Tabela 2. Análise acústica: distribuição dos dados demográficos da $\mathrm{F}_{0}$, divididos de acordo com o sexo, em relação aos diferentes tipos de disartrias

\begin{tabular}{|c|c|c|c|c|c|c|}
\hline \multicolumn{2}{|c|}{ Tipos de disartria } & \multicolumn{5}{|c|}{$\mathrm{F}_{0}$} \\
\hline & & Média & DP & Mediana & Mínima & Máxima \\
\hline \multirow[t]{2}{*}{ Flácida } & Homem & 139,17 & 38,69 & 136,37 & 94,97 & 188,98 \\
\hline & Mulher & 206,10 & 29,03 & 195,33 & 185,19 & 248,55 \\
\hline \multirow[t]{2}{*}{ Espástica } & Homem & 153,79 & 20,91 & 153,79 & 139,00 & 168,57 \\
\hline & Mulher & 199,74 & 33,54 & 205,13 & 159,32 & 229,38 \\
\hline \multirow[t]{2}{*}{ Hipocinética } & Homem & 138,39 & 26,82 & 136,15 & 109,24 & 180,41 \\
\hline & Mulher & 209,03 & 64,56 & 222,37 & 97,42 & 295,79 \\
\hline \multirow[t]{2}{*}{ Hipercinética } & Homem & 213,06 & 130,10 & 213,06 & 121,06 & 305,05 \\
\hline & Mulher & 190,61 & 29,50 & 193,27 & 141,15 & 214,36 \\
\hline \multirow[t]{2}{*}{ NMSU } & Homem & 165,48 & 42,92 & 188,58 & 116,76 & 210,51 \\
\hline & Mulher & - & - & - & - & - \\
\hline \multirow[t]{2}{*}{ Mista } & Homem & - & - & - & - & - \\
\hline & Mulher & 195,57 & 10,94 & 195,57 & 187,83 & 203,30 \\
\hline
\end{tabular}

Legenda: NMSU = Neurônio motor superior unilateral

Tabela 3. Análise acústica: distribuição dos dados demográficos de Jitter em relação aos diferentes tipos de disartrias

\begin{tabular}{lccccc}
\hline Tipo de disartria & \multicolumn{5}{c}{ Jitter } \\
\cline { 2 - 6 } & Média & DP & Mediana & Mínima & Máxima \\
\hline Flácida & 0,74 & 1,04 & 0,38 & 0,08 & 3,21 \\
Espástica & 0,73 & 0,58 & 0,71 & 0,14 & 1,43 \\
Hipocinética & 0,52 & 1,14 & 0,18 & 0,06 & 4,45 \\
Hipercinética & 1,39 & 2,35 & 0,40 & 0,11 & 6,59 \\
NMSU & 0,17 & 0,09 & 0,18 & 0,09 & 0,30 \\
Mista & 0,23 & 0,13 & 0,23 & 0,14 & 0,32 \\
\hline
\end{tabular}

Legenda: $\mathrm{NMSU}=$ Neurônio motor superior unilateral
Tabela 4. Análise acústica: distribuição dos dados demográficos de Shimmer em relação aos diferentes tipos de disartria

\begin{tabular}{lccccc}
\hline Tipo de disartria & \multicolumn{5}{c}{ Shimmer } \\
\cline { 2 - 6 } & Média & DP & Mediana & Mínima & Máxima \\
\hline Flácida & 7,63 & 5,70 & 4,62 & 3,61 & 16,66 \\
Espástica & 7,85 & 3,33 & 7,76 & 3,27 & 12,13 \\
Hipocinética & 6,21 & 3,49 & 5,25 & 2,83 & 15,94 \\
Hipercinética & 11,51 & 8,41 & 12,43 & 3,17 & 27,91 \\
NMSU & 7,44 & 3,32 & 6,54 & 3,79 & 12,63 \\
Mista & 5,55 & 4,04 & 5,55 & 2,69 & 8,40 \\
\hline
\end{tabular}

Legenda: NMSU = Neurônio motor superior unilateral

Tabela 5. Comparação entre a análise auditiva e acústica (qualitativa e quantitativa)

\begin{tabular}{|c|c|c|c|c|c|c|c|}
\hline \multirow[t]{2}{*}{ Análise auditiva } & & \multicolumn{6}{|c|}{ Análise acústica } \\
\hline & & Subharmônicos & $\begin{array}{l}\text { Definição dos } \\
\text { harmônicos }\end{array}$ & $\begin{array}{l}\text { Ruído entre os } \\
\text { harmônicos }\end{array}$ & Jitter & GNE & $\mathrm{F}_{0}$ \\
\hline \multirow[t]{3}{*}{ Tipo de voz } & Rouca & $p=0,445$ & $p=0,241$ & $p=0,665$ & $p=0,496$ & $p=0,577$ & \\
\hline & Soprosa & $p=0,655$ & $\mathrm{p}=0,040^{*}$ & $p=0,616$ & $p=0,048^{*}$ & $p=0,469$ & \\
\hline & Trêmula & & & & $p=0,678$ & & \\
\hline \multirow[t]{2}{*}{ Pitch } & Feminino & & & & & & $p=0,884$ \\
\hline & Masculino & & & & & & $p=0,931$ \\
\hline
\end{tabular}

${ }^{*}$ estatisticamente significante $(p<0,05)$ 
trabalham com voz estejam alertas para mudanças vocais sutis e para reconhecer suas implicações. Para pacientes com distúrbios do movimento, dados acústicos podem ser obtidos facilmente, de forma não invasiva e relativamente sem altos custos.

Quando temos dúvida sobre os dados acústicos utilizados, ou ainda, quando todos os outros métodos de avaliação falham, confiamos no poder de análise do nosso ouvido, que nos fornece, além de dados anatomofisiológicos importantes, informações sobre o grau de desagradabilidade da voz que está sendo avaliada. Desta forma, a análise acústica é um importante instrumento de avaliação, associada à análise perceptivo-auditiva.

Observamos que nas disartrias, há uma concomitância de diferentes tipos de voz num mesmo tipo de disartria, o que de certo modo impossibilita uma análise acústica totalmente confiável. O sinal acústico característico possibilita a análise visual do traçado; no entanto, mensurações não são confiáveis. A aperiodicidade das vozes das disfonias neurológicas pode impedir o uso de uma análise computadorizada automática, que analisa segmentos mais periódicos. Este tipo de sinal é justamente o que mais necessita de apoio tecnológico para a compreensão do resultado vocal. Mesmo não tendo uma medida acústica confiável, é importante quantificar e classificar diferentes realidades vocais. O mais importante das mensurações são as conclusões que conseguimos tirar sobre fisiologia laríngea e o raciocínio clínico que ela nos permite fazer.

A maioria da nossa amostra foi composta por disartria hipocinética (33,3\%). Estima-se que $89 \%$ dos pacientes com Doença de Parkinson experimentarão alterações vocais com a progressão da doença ${ }^{(11)}$, apresentando incidência significativa de alterações de voz e fala. Neste estudo, pôde-se observar que os pacientes que estavam em lista de espera já apresentavam instaladas as alterações de voz e fala.

A disartria, na Doença de Parkinson, pode ser caracterizada por monotonia de pitch e loudness, redução no estresse das palavras, imprecisão de consoantes e voz rouco-soprosa ${ }^{(12)}$. O uso de levodopa para recolocação de concentração de dopamina no núcleo estriado parece melhorar a articulação, qualidade vocal e variação de pitch.

Alguns pacientes parkinsonianos apresentam complicações a longo-prazo com o tratamento por levodopa, optando muitas vezes por submeter-se à cirurgia - Palidotomia Pós-ventral. Um interessante estudo ${ }^{(13)}$ foi realizado com o objetivo de avaliar a voz, por meio da analise acústica, de um paciente pré e pós a cirurgia. O estudo evidenciou uma melhora no Shimmer e nas medidas de ruído.

Em relação à análise auditiva, apresentada na Tabela 1, em que observamos as características perceptivas da voz nos diferentes tipos de disartria, nossos achados se aproximam muito aos encontrados na literatura ${ }^{(14-16)}$. No entanto, também podem ser encontrados voz trêmula e rouquidão para disartria hipocinética e soprosidade para disartria hipercinética, além de aspereza para Disartria do Neurônio Motor Superior Unilateral (NMSU) ${ }^{(17)}$. Cabe ressaltar que a avaliação subjetiva da intensidade vocal se deu a partir de gravação e, embora o controle do volume tenha sido considerado, não é uma medida confiável. Um dado mais fidedigno poderia ter sido uma análise objetiva da intensidade vocal com um medidor de nível sonoro ${ }^{(18)}$.
Nesta pesquisa, observamos que o tipo de voz mais presente nas disartrias foi a rouca e soprosa e, muitas vezes, essas duas combinações, voz rouco-soprosa. Na literatura ${ }^{(17,19)}$, encontra-se que os principais tipos de voz que caracterizam os pacientes neurológicos são a voz áspera e a voz rouca e, por vezes, pode ser observada "rouquidão molhada", um tipo de voz que além da irregularidade vocal, permite ouvir a característica molhada por estase de saliva sobre as pregas vocais ou nos seios piriformes. Podemos também observar soprosidade constante ou ocasional, emissão de característica comprimida, tensa-estrangulada, falhas na produção vocal e presença de tremor rápido - flutter $^{(19)}$.

Ainda em relação à análise auditiva, pôde-se observar traçado espectrográfico instável (acústica), fato que se deve possivelmente à fraqueza, lentidão ou incoordenação da musculatura laríngea comumente presentes nas disfonias neurológicas.

A avaliação global do traçado espectrográfico traz uma informação detalhada sobre a fonte sonora e o sistema de ressonância, representando uma verdadeira reprodução gráfica da qualidade vocal ${ }^{(19)}$. Sendo assim, observou-se neste trabalho que o traçado, além de se mostrar instável, apresentou-se em sua maioria sem harmônicos superiores, o que demonstra uma irregularidade na distribuição de energia no trato vocal, estando concentrada na cavidade nasal ou na região laringo-faríngea.

Em relação às medidas qualitativas da análise acústica, observamos que o traçado espectrográfico em todos os tipos de disartria apresentou-se instável. Quanto às variáveis acústicas quantitativas, apresentadas nas Tabelas 2, 3 e 4, verificamos que não houve diferença estatisticamente significante da freqüência fundamental entre os sexos, nas diferentes disartrias (flácida $-\mathrm{p}=0,033$; espástica $-\mathrm{p}=0,161$; hipocinética - $\mathrm{p}=0,016$; hipercinética $-\mathrm{p}=0,692$ ). Alguns autores referem que a análise acústica do pitch e loudness ilustram aspectos das alterações vocais que são dificilmente detectadas pelo ouvido humano ${ }^{(20)}$. Sendo assim, nesta pesquisa observou-se que o correlato acústico do pitch, ou seja, as médias de frequiência fundamental mostraram-se semelhantes entre os sexos nos diferentes tipos de disartria. No entanto, quando se trata da $\mathrm{F}_{0}$ mínima e máxima, observa-se que há uma disparidade muito grande, fato que se deve provavelmente por se tratar de um grupo muito heterogêneo e de características vocais diversas, em relação aos diferentes graus e tipos de disartria. Em estudo prévio $^{(21),} 83$ pacientes diagnosticados com miastenia gravis foram estudados e observou-se função fonatória anormal, sendo que a f0 aumenta nos pacientes com miastenia gravis generalizada com o avanço da doença. Sendo assim, os autores concluem que a análise acústica é um importante método de diagnóstico e avaliação para essa população.

Quanto às variáveis ciclo-a-ciclo, tais como Jitter e Shimmer, apresentadas nas Tabelas 3 e 4, observamos que em todos os tipos de disartria elas se mostraram aumentadas, o que concorda com a literatura que refere que essas medidas podem estar aumentadas nas disfonias neurológicas, pois relatam medidas de regularidade de vibração das pregas vocais, possivelmente reflexo da redução do controle neuromuscular do mecanismo laríngeo adutor e abdutor ${ }^{(19)}$. Ainda, a rouquidão pode refletir nas medidas acústicas de Jitter, como reflexo 
da assimetria de hipotonia laríngea, conduzindo a diferenças entre a tensão das pregas vocais ou vibração de massa, com subseqüente oscilação irregular de pregas vocais.

Embora as medidas ciclo-a-ciclo (Jitter e Shimmer) possam oferecer informações valiosas sobre instabilidades fonatórias, estudos de doenças neurológicas da voz requerem quantificação de instabilidades fonatórias de longo-prazo. Por exemplo, os segmentos de baixa frequiência nas vozes dos pacientes com Doença de Hungtinton e a amplitude de tremor observada nos parkisonianos, podem prover valiosas informações sobre a patofisiologia neurolaríngea que pode não ser aparente nas medidas ciclo-a-ciclo ${ }^{(17)}$. Além disso, cabe ressaltar que as medidas de perturbação, Jitter e Shimmer referem-se a quanto um determinado período de vibração diferencia-se do outro que o sucede, respectivamente quanto à frequiência e amplitude, ou seja, representam o nível de estabilidade vibratória e dependem do controle sobre o sistema fonatório. No entanto, sabemos da validade relativa das medidas de Jitter e Shimmer, investigadas no presente estudo, visto que uma laringe com alterações, seja pela presença de massa, seja por distúrbios no controle neuromuscular, produz mais ciclos erráticos que uma laringe normal $^{(6)}$. Desta forma, sabemos que as medidas de Jitter e Shimmer são mais importantes e mais relevadas na avaliação de vozes levemente alteradas.

Pudemos perceber, a partir dos dados apresentados na Tabela 5, que a comparação entre os achados das análises auditiva e acústica, em sua maioria, não foi significante, ou seja, não houve uma relação direta entre os achados subjetivos e os dados objetivos. A análise auditiva é soberana e não se pode esperar uma correlação direta entre essas análises, por exemplo, esperar pitch agudo sempre que se encontra $\mathrm{F}_{0}$ aumentada. Vale ressaltar que a análise acústica não sobrepuja a avaliação perceptivo-auditiva, sendo complementar a mesma. Desta forma, é um poderoso instrumento para o detalhamento da função vocal, permitindo uma maior compreensão do problema do paciente, facilitando o raciocínio clínico para o diagnóstico da disfonia.

Houve diferença estatisticamente significante entre voz soprosa e Shimmer alterado $(\mathrm{p}=0,048)$ e entre a definição dos harmônicos e voz soprosa $(\mathrm{p}=0,040)$, sendo assim, observou-se correlação entre a presença de ruído à emissão e soprosidade.
Quando se tem soprosidade na voz, observa-se presença de ruído no espectrograma que se traduz, por má definição dos harmônicos, os quais são substituídos por ruído. O Shimmer altera-se principalmente nas situações de redução da resistência glótica, presença de lesões de massa nas pregas vocais e correlaciona-se com a presença de ruído à emissão ${ }^{(6)}$. Sendo assim, a associação entre soprosidade e Shimmer foi direta, então quando auditivamente observa-se soprosidade, esperase que na análise acústica as medidas de Shimmer estejam alteradas, no caso, aumentadas.

Estudos como este permitem considerações teóricas e de caráter prático no estudo da fonação de pacientes com doenças neurológicas. A descrição subjetiva (avaliação perceptivoauditiva) e objetiva (análise acústica) realizadas neste trabalho representam apenas uma parte da imensa gama de assuntos que ainda devem ser explorados em relação à contribuição da análise acústica para pacientes disártricos.

Ao identificar os parâmetros alterados a partir de uma análise vocal completa (auditiva e acústica), pode-se ter compreensão total dos aspectos fonatórios das disartrias. Esta análise é de crucial valor no planejamento terapêutico, pois, às vezes, a alteração isolada de um parâmetro, por exemplo, a redução da intensidade vocal, pode limitar em grande grau a comunicação funcional do indivíduo. Tal percepção irá auxiliar para o diagnóstico precoce e na escolha do tratamento para que o paciente tenha uma melhor qualidade de vida, tendo sua comunicação não tão prejudicada.

\section{CONCLUSÃO}

A partir da comparação entre os dados das análises perceptual auditiva e acústica observamos que não houve equivalência para todos os parâmetros analisados. Este dado sugere que as duas análises devam ser utilizadas de forma conjunta na avaliação clínica das disartrias.

\section{AGRADECIMENTOS}

Agradecemos à FAPESP, o apoio financeiro para a realização deste estudo.

\begin{abstract}
Purpose: To compare data found in auditory-perceptual analyses (subjective) and acoustic analyses (objective) in dysarthric patients. Methods: Forty-two patients with well defined neurological diagnosis, 21 male and 21 female, were evaluated in auditory-perceptual parameters and acoustic measures. All patients had their voices recorded. Auditory-perceptual voice analyses were made considering type of voice, resonance (balanced, hipernasal or laryngopharyngeal), loudness (adequate, decreased or increased), pitch (adequate, low or high), vocal attack (isochronic, sudden or breathy), and voice stability (stable or unstable). Acoustic analyses were made with GRAM 5.1.7 Program that considered voice quality and spectrographic tracing, and Vox Metria Program to obtain objective measures. Results: The comparison between auditory-perceptual and acoustic data showed no correlation for all the parameters analyzed. It was found a significant difference between breathiness and shimmer alteration $(\mathrm{p}=0.048)$, and between breathiness and harmonics definition ( $\mathrm{p}=0.040$ ), evidencing correlation between noise presence during emission and breathiness. Conclusion: Acoustic analysis associated to auditory-perceptual analysis provided different but complementary data, helping the clinical diagnosis of dysarthias.
\end{abstract}

Keywords: Dysarthria; Voice disorders; Speech acoustics; Speech; Voice quality 


\section{REFERÊNCIAS}

1. Darley FL, Aronson AE, Brown JR. Differential diagnostic patterns of dysarthria. J Speech Hear Res. 1969;12(2):246-69.

2. Oliveira CR, Ortiz KZ, Vieira MM. Disartria: estudo da velocidade de fala. Pró- Fono. 2004;16(1):39-48.

3. Griffiths C, Bough D. Neurologic diseases and their effect on voice. J Voice. 1989;3(2):148-56.

4. Urban PP, Rolke R, Wicht S, Keilmann A, Stoeter PC, Hopf HC, Dieterich M. Left-hemispheric dominance for articulation: a prospective study on acute ischaemic dysarthria at different localizations. Brain. 2006;129(Pt 3):767-77.

5. Abberton E. Phonetic considerations in the design of voice assessment material. Logoped Phoniatr Vocol. 2005;30(3-4):175-80.

6. Behlau MS, organizadora. A voz do especialista. Rio de Janeiro: Revinter; 2005. v.2. p.11-186.

7. Behlau MS, organizadora. Voz: o livro do especialista. Rio de Janeiro: Revinter; 2001.

8. Eadie TL, Doyle PC. Classification of dysphonic voice: acoustic and auditory- perceptual measures. J Voice. 2005;19(1):1-14.

9. Pützer M, Wokurek W. [Multiparametric description of voice quality for normal male and female voices based on acoustic analyses]. Laryngorhinootologie. 2006;85(2):105-12. German.

10. Awan SN, Roy N. Acoustic prediction of voice type in women with functional dysphonia. J Voice. 2005;19(2):268-82.

11. Azevedo LL, Cardoso F, Reis C. Análise acústica da prosódia em mulheres com doença de Parkinson: comparação com controles normais. Arq Neuropsiquiatr. 2003;61(4):999-1003.
12. Pinto S, Ozsancak C, Tripoliti E, Thobois S, Limousin-Dowsey P, Auzou P. Treatments for dysarthria in Parkinson's disease. Lancet Neurol. 2004;3(9):547-56

13. Mourão LF, Aguiar PMC, Ferraz FAP, Behlau MS, Ferraz HB. Acoustic voice assessment in Parkinson's disease patients submitted to posteroventral pallidotomy. Arq Neuropsiquiatr. 2005;63(1):20-5.

14. Medeiros ME. Disatrofonia: uma visão fonoaudiológica. Fonoaudiol Brasil. 1999;2:27-37.

15. Kent RD, Kim YJ. Toward an acoustic typology of motor speech disorders. Clin Linguist Phon. 2003;17(6):427-45.

16. Carrillo L, Ortiz KZ. Análise vocal (auditiva e acústica) nas disartrias. Pró-Fono. 2007;19(4):381-6.

17. Duffy JR. Motor speech disorders: substrates, differential diagnosis, and management. 2nd ed. St. Louis: Mosby; c2005.

18. Carrilo L. Efeito da técnica de sobrearticulação em alunos de teatro, TV e cinema [monografia]. São Paulo: Centro de Estudos da Voz; 2005.

19. Jiang J, Lin E, Hanson DG. Acoustic and airflow spectral analysis of voice tremor. J Speech Lang Hear Res. 2000;43(1):191-204.

20. Samuel C, Louis-Dreyfus A, Couillet J, Roubeau B, Bakchine S, Bussel B, Azouvi A. Dysprosody after severe closed head injury: an acoustic analysis. J Neurol Neurosurg Psychiatry. 1998;64(4):482-5.

21. Sun W, Liu H, Wan X, Cong Z, Yang H, Sun Y. [The study of phonatory function in myasthenia gravis patients]. Lin Chuang Er Bi Yan Hou Ke Za Zhi. 2005;19(15):685-7. Chinese. 